\title{
Interactive model-based decision-making tools in early product platform design
}

\author{
Iñigo Alonso Fernandez ${ }^{1}$, Massimo Panarotto ${ }^{1}$, Ola Isaksson ${ }^{1}$ \\ ${ }^{I}$ Chalmers University of Technology \\ inigo.alonso@chalmers.se \\ massimo.panarotto@chalmers.se \\ ola.isaksson@chalmers.se
}

\begin{abstract}
Integrating new technologies in existing product platforms presents challenges not only when the decision of going ahead with the integration is taken, but also in the earlier design of the platform structure to accommodate hypothetical changes in the future. Common heuristics do no guarantee that the optimum solution can be found to these kinds of problems, and biases lead to systematic distortions in decision-making. Additionally with the global zeitgeist around sustainable development, decision makers will increasingly ask for paths to many different versions of success, not just the traditional profit maximization one. A set of common models that accompanies the product platform all through its lifecycle to support decision makers can enable better fulfilment of the expectations of all stakeholders. But it is difficult to unify and objectively gather the views of multiple stakeholder simultaneously. An interactive modelbased decision making support system is proposed as a tool to solve the mentioned challenges. In this paper we describe and experiment with the main technological foundations of such a tool. These include an web-based front end, and a real-time NoSQL database in the back end. The client web application (webapp) enables user inputs, runs quantitative models, and visualizes results. The database records results and enables the use of common inputs and common visualization of the results. The models that run directly in the client are developed offline and can be continuously deployed with no downtime for concurrent users. The technology stack used demonstrates that rapid prototyping of tools using state-of-the-art web technologies provides quick results and enables researchers to make quick iterations that can be easily deployed in industrial use cases. The presented method is a new approach to providing digital support to the design process, by enabling better informed decisions during the product development process early phases. In this paper, an introduction and background to the problem and current state of the art is summarized, a method to approaching the topic is described, an experiment performed in front of a life audience is presented, and hints for future developments are considered in the discussion and conclusion sections.
\end{abstract}

Keywords: decision making, digital design, model based engineering, design support system 


\section{Introduction}

Model-based (sometimes referred as model-driven in the software domain) approaches to supporting decision-making in early design phases have been proposed in the past (Wall, Bertoni, \& Larsson, 2018). But previous tools depended on legacy systems (e.g. Microsoft Excel, MATLAB, Java-based applications), or introduced new locally installed software. The approach we propose in this paper is to use state-of-the-art frameworks that result in not only better-looking user interfaces, but also leverage the exiting pool of talent in web development worldwide. This approach builds on open and modular principles of conducting research, and aims to develop tools, models, and results, and to bringing research projects more quickly into utilization as they build on open standards and conventions widely shared. The other forward looking goal is to support the analysis of product platforms in their early development phases.

This paper demonstrates the use of modern web software frameworks, that help minimize the introduction of new software (everything runs in a browser) into any industrial or academic environment. This work was performed in the context of the Value and flexibility Impact analysis for Sustainable Production (VISP) project, and shares its focus on product platform modelling. One challenge that needs to be addressed is to combine companies desire to comply with internal software environments, while being generally applicable also in extended enterprises.

The models will be used to support decision making during product platform design, and support exploration of platform strategies and trade-offs when e.g. considering technology integration or replacement. A Service Oriented Architecture (SOA) approach to the development of inter-compatible components that use web Application Programming Interfaces (APIs) inside research groups will enable more collaboration not only between individual contributors, but also among groups distributed worldwide. A downside of the technological choices made in this approach is that the learning curve is steeper and the background of the researchers plays a major role in how intuitive the concepts are.

Table 1 summarizes the alignment of the general methodology proposed and the experiment discussed in sections 3 and 4 .

Table 1 Aspects of the methodology studied in the experiment

Methodology aspects

Model-based

Interactive collaboration

Decision-making support

Constraint-free data modelling

Architecture structure

Connection between the product structure

and value attributes

Architecture structure metrics (flexibility, complexity...)

\section{Experiment characteristics}

Models defined offline, running in the user client

Webapp with real-time database backend Simple product configurator with go/no go approval

NoSQL document database without predefined schema

$\mathrm{n} / \mathrm{a}$

$\mathrm{n} / \mathrm{a}$

$\mathrm{n} / \mathrm{a}$ 


\section{Background}

In this section we present a summary of the background on product platforms, decision making within the design process and the use of models, highlighting some well supported approaches that nevertheless could be updated or extended.

\subsection{Product platforms}

Ulrich (1995) defined product architecture as:

(1) the arrangement of functional elements,

(2) the mapping from functional elements to physical components (also referred as technical solutions) and

(3) the specification of the interfaces among interacting system components.

Or in a less formal summary: "the scheme by which the function of a product is allocated to physical components". Building further upon Ulrich definition, a product platform can be defined as a product architecture from which a set of variants can be derived, usually in order to reduce manufacturing and procurement costs. These definitions support the idea of using graph representations of the data involved.

Complexity makes the analysis of product platforms much more difficult than in the case of individual products. At the same time, there is a need to accommodate changes that range from incremental to breakthroughs, while navigating a lot of ambiguity, and trying to minimize the failure rate (and still be able to mitigate the consequences of decisions that turned out worse than anticipated). In the case of exiting product portfolios that need to be maintained, procedural models have shown promise in keeping valid sets of portfolio rules across the whole organization (Braun, Kreimeyer, \& Paetzold, 2018).

Many methods are available in the literature to manage the development of modular product families, among them the Life phases Modularization (Krause et al., 2014) is of interest, as it aligns the modules structure to the different temporal phases of the product's lifecycle, making the coordination of the family structure more clear.

\subsection{Design process and decision making}

Jonassen (2008) described design as an iterative process of decision-making. As such, it is a demanding exercise in problem solving. As the process iterations advance, the degrees of freedom available to the designers decrease as decisions are taken. The decider can be an individual for some decisions, and whole decision team with its steering group, outside experts and implements for others. Either way the process around each of the decisions can be summarized as:

1. Framing the problem and the current situation

2. Create real alternatives within the constraints of the current world status

3. Assess and evaluate those alternatives on different criteria, assigning different weights to the criteria

4. Visualize real and tangible trade-offs between the alternatives

5. Make decision

6. Monitor the outcomes and consequences to evolve the models and the understanding of the new situation 
The use of models for decision making during the design process is illustrated in Figure 1, by complementing the process described by Jonassen (vide supra). The expectations and needs of the stakeholders determine the engineering aspects that are incorporated into mathematical models. These models are used determine the situation in different scenarios and the results are visualized to enable informed decisions. In this step the users can question the fundamentals of the model and of the scenario selection and successive iterations may be performed. The impact of the decisions could in the future be tracked and compared to the fulfillment of the original expectations for validation of the process.

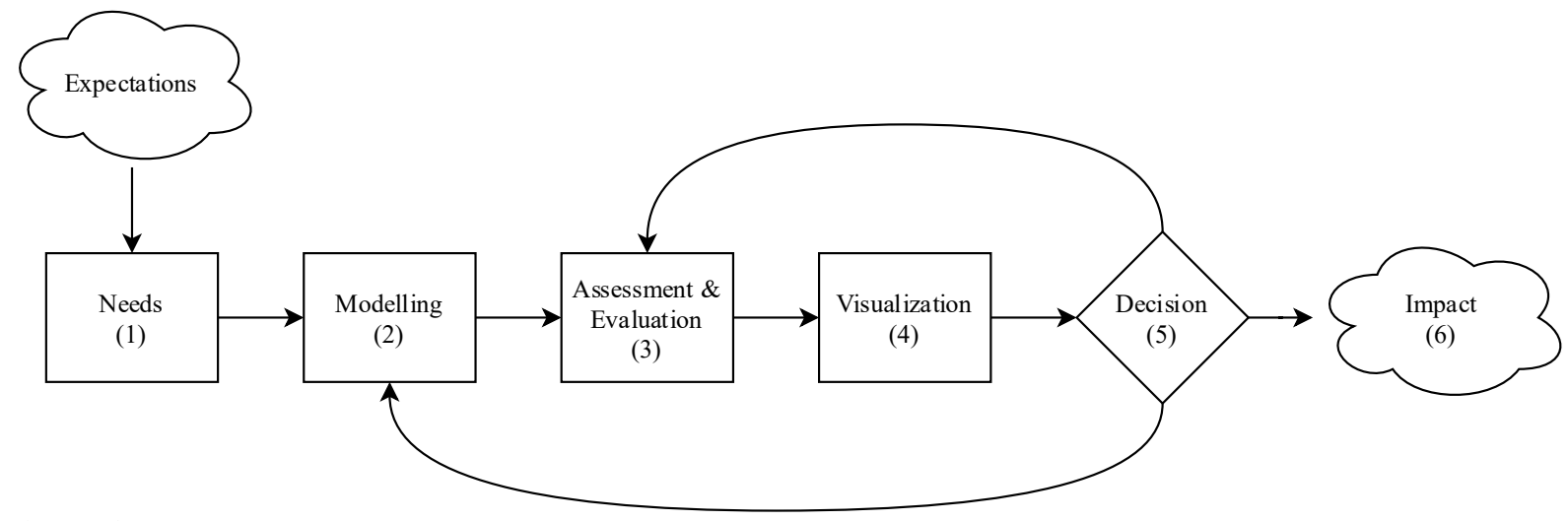

Figure 1. Model use process.

For the development of individual products, Agile approaches have been studied and their ability to track objectives along the entire product development process have been validated (Albers et al., 2018). The $\mathrm{ACD}^{3}$ (Activity-Centered Design)-matrix has also shown potential to steer the design process by clearly highlighting the design parameters that need to be examined and determined during the development (Bligård, Simonsen, \& Berlin, 2018).

Decisions and associated trade off discussions regarding technology integration in platforms (Alonso Fernández, Panarotto, \& Isaksson, 2020) for complex products are often made in industry by gathering stakeholders (including the decision markers) in large meetings. They represent different views and knowledge domains, but representing several views simultaneously requires tedious iterations to reach a shared understanding. Enabling interactive situations, allowing the decision-makers to interact with the underlying data and validated models that synthesize multiple perspectives, is a powerful technique to improve decision quality, where engineering modelling results have been prepared, and prepared for "interactiveness". This is studied in e.g. Visual Analytics domains, see e.g. (Hettenhausen, Lewis, Randall, \& Kipouros, 2013) and (Kipouros \& Isaksson, 2014).

Decision-making during the cooperative design of a modular product family has been further studied recently by Windheim, (2020), highlighting the positive effect of interactivity during the actual decision-making meeting. An approach based on interactive dashboard allows for straightforward discussion and explanation of the alternatives and trade-offs present, as well as the different levels and models utilized.

\subsection{Models}

The parties participating in the decision process should share a common understanding of several models representing the current circumstances:

- Product Architecture: functions, components and their relations

- Engineering Performance: attributes of the behavior during operation 
- Product Value: positive impact in fulfilling the stakeholder expectations

- Market Demand: as indication of the potential sales

- Manufacturing Cost: calculating the variable costs

- Investment/Finance: aggregation of the economic metrics and context

These types of models needed to translate product family design decisions into profitability for a company are summarized in Figure 2.

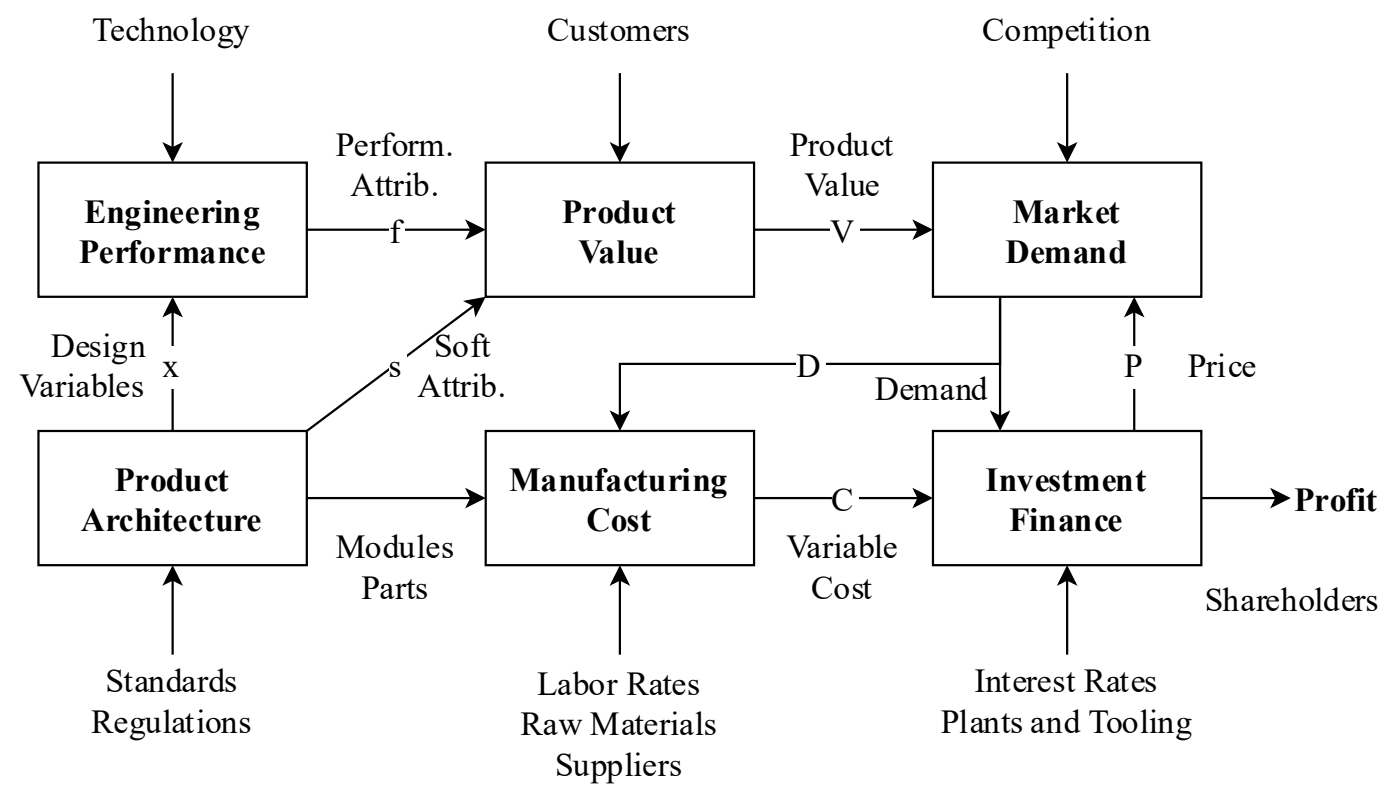

Figure 2. Single product reasoning and modelling framework, adapted from de Weck, (2006).

The target platform structure, where the relation between the components/modules and the functions the platform-derived products are defined, can be expressed in many different forms. Often, the main application domain sets the basis for the management of the assets and artifacts of the product platform like e.g. Electronic Computer-Aided Design (ECAD), Electronic Bill Of Materials (EBOM), Product Lifecycle Management (PLM). Hackl and Krause (2017) modelled the interrelations between product structures and their effects. As modern products are multi-technological, it is important to support multiple technologies from different domains and their interdependencies.

The Enhanced Function-Means (EF-M) method of functional decomposition is an option very well suited for the description of the platform in abstract form, enabling alternatives generation and selection on a pure functional level, while considering existing constraints (Müller et al., 2019).

The Technology input could be managed by using e.g. Technology Maps (Zavareh, Sadaune, Siedler, Aurich, \& Klaus, 2018) or other technology evaluations processes (Mämmelä et al., 2018). Key performance Indicators have been shown to support the development of product families (Schmidt, Schwöbel, \& Lienkamp, 2018). The manufacturing costs are usually included in the model of the production system. When the production system is modelled in a Product Development Process (PDP), it is usually included quite late in process (Henriksson \& Detterfelt, 2018). Despite the popularity of agile methods integrated processes with product and production system development are not very well developed in the literature. 
Sustainability is a topic that traditionally was pursued following the inputs of environmental regulations, but as awareness among consumers increase, it is becoming a selling point, adding customer value. Another topic that is gaining traction in last decades is the "servilization" of products into Product Service Systems (PSS). Isaksson, Hallstedt, \& Rönnbäck (2018) proposed incorporating both these trends into digital models to support the product development process.

When following a platform strategy, the organization will have to source inputs by conducting market research, managing the supply chain, analyzing macroeconomic trends, actively managing customer relationships, being involved in the Research and Development (R\&D) community, and developing its own people, among other activities. These inputs will not only feed the models, but also serve as initiators of changes to evolve the models, based on measurements and empirical evidence, rather than opinions.

Models might be too time consuming to develop in a timely matter when a decision is needed. Additionally they present a natural trade-off between their accuracy and their tractability. To be able to understand them in a more intuitive way, we propose the use of interactive models, developed in parallel to all other design activities and acting as black boxes during the evaluation and visualization phases, but providing instant feedback to the decision makers. The use of software alleviates the burden of dealing with complexity, but computer models needs good validation during their development. Building the model can also serve as a way to better understand the system. Matthiesen \& Nelius (2018) found evidence that verifying assumptions on a system's function and behavior enhances the completeness and correctness of the analysis of technical systems.

\section{Method}

Web-based approaches have been explored for product design for decades, including early experiments in interactive design and visualization (Lau, Mak, \& Lu, 2003). The buzzword "web 2.0" from previous decades has been superseded by other concepts like the "social web", the "Internet of Things (IoT)", and "Industry 4.0". New paradigms in web development, such as "serverless", "Single Page Application (SPA)", "Cloud computing", "continuous integration" and "API first design" are gaining traction. The application of these new technological capabilities to the development of product platforms, such as at a car or a truck manufacturer, are expected to provide more insightful decision support tools.

To conduct an experiment on the rapid development of a tool that could be used in an interactive decision-making environment where many stakeholders are present, we used a JavaScript (js) framework to build a SPA, a real-time document database to store data, and deployed the application on a cloud computing hosting platform with continuous deployment directly from a git repository.

The architecture of the deployment system is illustrated in Figure 3. A researcher and their local environment is represented in the lower left corner. The code is version controlled using a git repository, also hosted remotely at the GitHub cloud. From there the latest master branch of the code is continuously deployed to the Netlify cloud for hosting of the static assets of the SPA. Both the local environment and any remote user accessing the cloud hosted instance has direct access to the Cloud Firestore database. The users and the setup of the use case described in the next section are represented in the top right corner of the figure. 


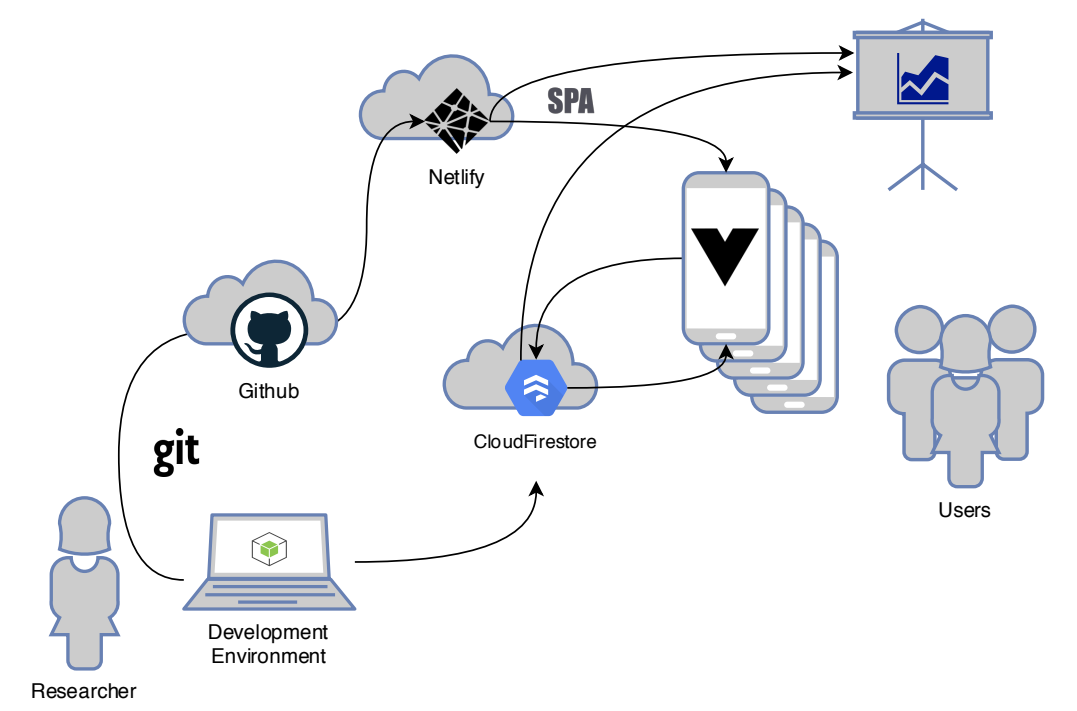

Figure 3. Cloud deployment diagram.

Product family design challenges like high dimensionality and computational complexity have been explored (Simpson, Jiao, Siddique, \& Hölttä-Otto, 2014) by using spreadsheets and the Applied Research Laboratory's Trade Space Visualizer (ATSV), a Java-based application (Stump, Lego, Yukish, Simpson, \& Donndelinger, 2007). The need to integrate a diverse set of data and data sources calls for a more open and unconstrained approach to the early definition of entities and parameters. In this approach we utilize a NoSQL database, in particular a document database for small demonstrations and graph databases for further research. The EF$\mathrm{M}$ is particularly well suited to being represented in graph databases as a collection of vertices (Functional Requirement, Design Solution, Constraint) and edges (e.g. is solved by, requires function, interacts with).

\section{Experiment: Wingquist Laboratory Annual Seminar}

During the 2019 Wingquist Laboratory Annual Seminar ${ }^{1}$, all the research groups within the Wingquist Laboratory at the Chalmers University of Technology presented the latest results from their research to manufacturing industries. The seminar was well attended, with around a hundred people in the audience.

To demonstrate the scalability and intuitiveness of our approach, we performed an online experiment with an audience during the event. The audience got to interact with a simple tool that allowed the combination of choices, the visualization of impacts in various metrics (e.g. cost, performance, sustainability). The users were able to express their different perspectives on what a good design for a particular product (i.e. a kayak) would be in this configurator-like tool. The concept for the experiment extended previous work on support for the additive manufacturing of mass customized large-scale products (Lithgow et al., 2019).

The cloud-based web application, allowed several models to run directly on the client, as they were delivered as part of the SPA serving as frontend for the tool. The SPA was developed using the VUE.js framework, served from a Node.js instance. Screenshots of different parts of the configurator tool can be seen in Figure 4. The tool provided data management and visualization capabilities but lacked any Multidisciplinary Design Optimization (MDO) framework unlike previous research by (Papageorgiou \& Ölvander, 2018).

\footnotetext{
${ }^{1}$ https://www.chalmers.se/en/centres/wingquist/events/annual-seminar/Pages/Annual-Seminar-2019.aspx
} 

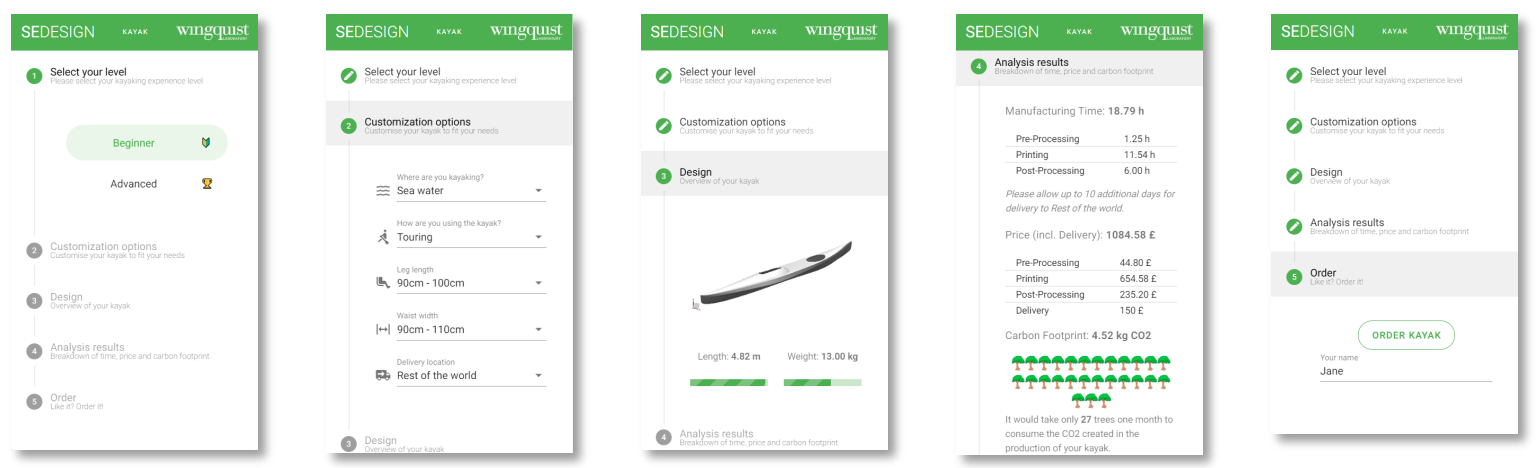

Figure 4. Screenshots of the web app in rendered in the mobile form-factor.

Quantitative models of the following kinds were included in the tool:

- Product Architecture: different geometries and features

- Engineering Performance: weight, handling in different conditions

- Product Value: different functionality depending on the customer experience

- Manufacturing Cost: fixed and variable costs

- Sustainability: $\mathrm{CO}_{2}$ emissions model

These models were selected to display general capabilities, despite not being of general interest for all possible stakeholders at the same time.

A cloud-based real time document database (Firebase Cloud Firestore) collected the data generated by the users, and streamed it to a summary page displayed during the presentation in a big screen, as shown in Figure 5. The audience was able to use their smartphones or laptops and start making decisions and visualizing results in a matter of seconds. In total 45 audience members submitted their results to the cloud database. The development of this experiment took around three days for a researcher with only hobbyist-level web programming background.

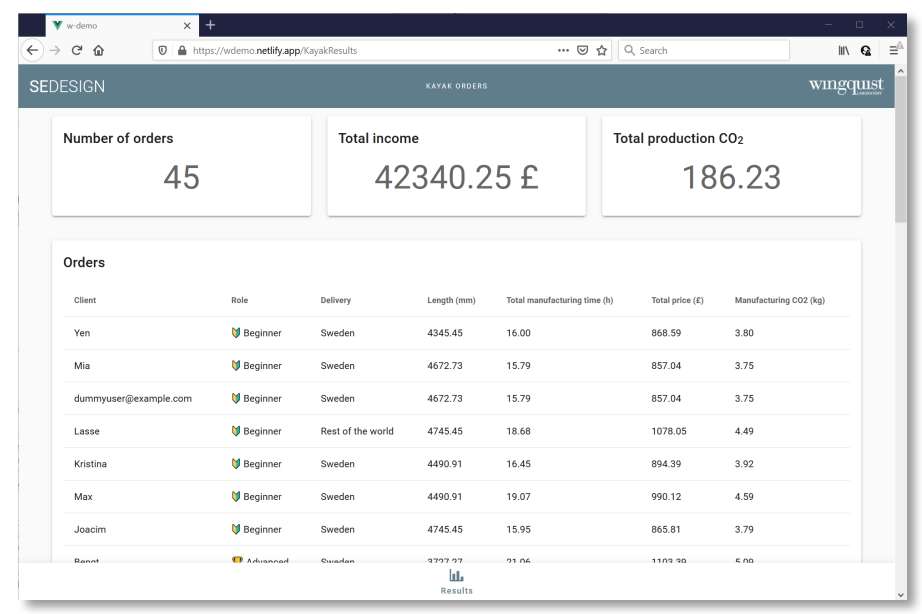

Figure 5. Screenshot of the summary view of real-time submissions.

\section{Discussion}

From a practitioner's point of view, having a plan that is sensible and within the realm of the possible can be much more desirable than a slow process of incremental improvements in the quality of the final solution. Existing methods such as matrix-based methods (Rapp, Moeser, Eichhorn, \& Albers, 2018) require some effort in their creation, but more importantly, require 
having a method to follow defined from the beginning. The problem with this approach is that the ways in which the implicit mental models embodied by the method constrain the designers only become apparent when compared to a different model, or categorization, or theory, i.e. they might reduce the design space inadvertently.

Starting the development of the framework with a schema-less strategy, on the other hand can devolve in a state of disarray. 'Garbage collection' utilities might be needed in the future to prune the graphs, in similar fashion to the automatic ontology-based integration and synchronization of the various sources of product documentation proposed by (Kügler, Schleich, \& Wartzack, 2018). The use of cloud-based solutions versus local implementations can pose data security risks, of great importance to industrial practitioners. This can be mitigated by running the same codebase locally by providing alternative databases (even inbrowser databases are available). The way forward includes the use of Progressive Web Application (PWA) concepts like these, with the added benefit of an even better user experience due to the integration with the client's operating system.

As summarized in Table 2, there are upsides to this approach, but also certain weaknesses.

Table 2 Strengths and weaknesses of the proposed approach.

\begin{tabular}{cc}
\hline Strengths & Weaknesses \\
\hline - Data-driven & $\circ$ Still rely on assumptions \\
- Minimization of subjectivity & $\circ$ Doesn't guarantee best decision \\
- Intuitive interface & $\circ$ Can overlook key aspects \\
- Fast turn-around into industrial use & $\circ$ Steep learning curve \\
- Vibrant technology ecosystem & $\circ$ 'Pioneers get shot in the back' \\
\hline
\end{tabular}

The proposed method of rapid application development helps in creating placeholders to represent aspects of some situation of interest during the design process to a range of stakeholders. This assists those stakeholders in achieving some purpose(s) relevant to them (as they might differ). The end goal is to enable practitioners to make explicit the implicit models of the situation that each of the stakeholders is operating under, as those are the source of most of the constraints that could be alleviated by a shared common model. Product development and strategic planning could benefit from a shared framework (Patel, Kalita, \& Asthana, 2018), more so in the case of product platforms. The future development of the proposed approach should consider integrating the scenario analysis, as seen in the concept of Value Creation Strategies (VCS) in the Value-Driven Design (VDD) methodology (Isaksson et al., 2013). A complementary feature to deal with uncertainty would be to include decision tree modelling, including estimates and probabilities to calculate the likelihood of possible outcomes, and thus the possible net gains to be obtained by following each of the branches.

A collaborative decision-making process is an interaction between multiple people in which each person's payoff is affected by the decisions made by others, i.e. a "game" in Game Theory. The interactions between stakeholders in the design process should be non-competitive, to avoid local optimization in individual silos. Sharing a virtual space where assumptions can be tested, and discussion can be backed with rational arguments could be of great support for decision making teams. 


\section{Conclusion}

The method presented in this paper is a new approach to providing digital support tools to the design process. Tools that can enable better informed decisions during the product development process, as the inputs of different stakeholders can be collected and unified in a coherent view. The experiments so far have shown that rapid iteration on the user interface and data models is possible with modern tools, and this results in very engaged stakeholders.

This approach could help managers in empowering designers to take action without having to seek approval for every decision. Further development of more sophisticated models and interfaces will be needed to validate the method on real industrial cases.

\section{Acknowledgement}

Financial support for this work was provided by the VISP project, co-funded by the Swedish Governmental Agency for Innovation Systems (VINNOVA), Volvo Cars Group (VCG) and Volvo Group Trucks Technology (VGTT).

\section{References}

Albers, A., Heimicke, J., Hirschter, T., Richter, T., Reiß, N., Maier, A., \& Bursac, N. (2018). Managing Systems of Objectives in the agile Development of Mechatronic Systems by ASD - Agile Systems Design. Proceedings of NordDesign: Design in the Era of Digitalization, NordDesign 2018.

Alonso Fernández, I., Panarotto, M., \& Isaksson, O. (2020). Identification of Technology Integration Challenges at Two Global Automotive OEMs. International Design Conference - DESIGN 2020. https://doi.org/https://doi.org/10.21278/idc.2020.0000

Bligård, L. O., Simonsen, E., \& Berlin, C. (2018). Perceived industrial usefulness of the ACD3matrix - an interview study with product developers. Proceedings of NordDesign: Design in the Era of Digitalization, NordDesign 2018.

Braun, F., Kreimeyer, M., \& Paetzold, K. (2018). Procedural model to ensure consistency and validity of complex, variant-oriented product portfolios. Proceedings of NordDesign: Design in the Era of Digitalization, NordDesign 2018.

de Weck, O. L. (2006). Determining Product Platform Extent. In T. W. Simpson, Z. Siddique, \& J. R. Jiao (Eds.), Product Platform and Product Family Design: Methods and Applications (pp. 241-301). https://doi.org/10.1007/0-387-29197-0_12

Hackl, J., \& Krause, D. (2017). Towards an impact model of modular product structures. Proceedings of the International Conference on Engineering Design, ICED, 3(DS87-3), 151-160.

Henriksson, F., \& Detterfelt, J. (2018). Production - as seen from product development: A theoretical review of how established product development process models address the production system. Proceedings of NordDesign: Design in the Era of Digitalization, NordDesign 2018.

Hettenhausen, J., Lewis, A., Randall, M., \& Kipouros, T. (2013). Interactive multi-objective particle swarm optimisation using decision space interaction. 2013 IEEE Congress on Evolutionary Computation, CEC 2013, 3411-3418. https://doi.org/10.1109/CEC.2013.6557988

Isaksson, O., Hallstedt, S. I., \& Rönnbäck, A. Ö. (2018). Digitalisation, sustainability and servitisation: Consequences on product development capabilities in manufacturing firms. Proceedings of NordDesign: Design in the Era of Digitalization, NordDesign 2018.

Isaksson, O., Kossmann, M., Bertoni, M., Eres, H., Monceaux, A., Bertoni, A., ... Zhang, X. (2013). Value-Driven Design - A methodology to Link Expectations to Technical 
Requirements in the Extended Enterprise. INCOSE International Symposium, 23(1), 803819. https://doi.org/10.1002/j.2334-5837.2013.tb03055.x

Jonassen, D. (2008). Instructional design as design problem solving: An iterative process. Educational Technology: The Magazine for Managers of Change in Education, 48(3), 2126.

Kipouros, T., \& Isaksson, O. (2014). Integrating value assessment into the computational engineering design cycle. OPT-i 2014 - 1st International Conference on Engineering and Applied Sciences Optimization, Proceedings, (June 2014), 2446-2455.

Krause, D., Beckmann, G., Eilmus, S., Gebhardt, N., Jonas, H., \& Rettberg, R. (2014). Integrated Development of Modular Product Families: A Methods Toolkit. In T. W. Simpson, J. (Roger) Jiao, Z. Siddique, \& K. Hölttä-Otto (Eds.), Advances in Product Family and Product Platform Design: Methods \& Applications (pp. 245-269). https://doi.org/10.1007/978-1-4614-7937-6_10

Kügler, P., Schleich, B., \& Wartzack, S. (2018). Consistent digitalization of engineering design - an ontology-based approach. Proceedings of NordDesign: Design in the Era of Digitalization, NordDesign 2018.

Lau, H. Y. K., Mak, K. L., \& Lu, M. T. H. (2003). A virtual design platform for interactive product design and visualization. Journal of Materials Processing Technology, 139(1-3 SPEC), 402-407. https://doi.org/10.1016/S0924-0136(03)00510-7

Lithgow, D., Morrison, C., Pexton, G., Panarotto, M., Müller, J. R., Almefelt, L., \& McLaren, A. (2019). Design Automation for Customised and Large-Scale Additive Manufacturing: A Case Study on Custom Kayaks. Proceedings of the Design Society: International Conference on Engineering Design, 1(1), 699-708. https://doi.org/10.1017/dsi.2019.74

Mämmelä, J., Juuti, T., Korhonen, T., Julkunen, P., Lehtonen, T., Pakkanen, J., \& Vanhatalo, M. (2018). Evaluating the value and costs of technology in the manufacturing industry. Proceedings of NordDesign: Design in the Era of Digitalization, NordDesign 2018.

Matthiesen, S., \& Nelius, T. (2018). Managing assumptions during analysis - study on successful approaches of design engineers. Proceedings of NordDesign: Design in the Era of Digitalization, NordDesign 2018.

Müller, J. R., Isaksson, O., Landahl, J., Raja, V., Panarotto, M., Levandowski, C., \& Raudberget, D. (2019). Enhanced function-means modeling supporting design space exploration. Artificial Intelligence for Engineering Design, Analysis and Manufacturing, 1-15. https://doi.org/https://doi.org/10.1017/ S0890060419000271 Received:

Papageorgiou, A., \& Ölvander, J. (2018). A data management and visualization tool for integrating optimization results in product development. Proceedings of NordDesign: Design in the Era of Digitalization, NordDesign 2018.

Patel, A., Kalita, P. C., \& Asthana, S. (2018). Design for future: Strategic planning and design innovation framework for digital organizations. Proceedings of NordDesign: Design in the Era of Digitalization, NordDesign 2018.

Rapp, S., Moeser, G., Eichhorn, P., \& Albers, A. (2018). Identifying expedient variations in PGE - Product generation engineering. Proceedings of NordDesign: Design in the Era of Digitalization, NordDesign 2018.

Schmidt, M., Schwöbel, J., \& Lienkamp, M. (2018). Developing key performance indicators for variant management of complex product families. Proceedings of NordDesign: Design in the Era of Digitalization, NordDesign 2018.

Simpson, T. W., Jiao, J. R., Siddique, Z., \& Hölttä-Otto, K. (2014). Advances in product family and product platform design: Methods \& applications. In Advances in Product Family and Product Platform Design: Methods and Applications. https://doi.org/10.1007/978-1-46147937-6

Stump, G., Lego, S., Yukish, M., Simpson, T. W., \& Donndelinger, J. A. (2007). Visual 
Steering Commands for Trade Space Exploration: User-Guided Sampling With Example. Proceedings of the ASME 2007 International Design Engineering Technical Conferences \& Computers and Information in Engineering Conference. Las Vegas, Nevada, USA: ASME.

Ulrich, K. T. (1995). The role of product architecture in the manufacturing firm. Research Policy, 24, 419-440.

Wall, J., Bertoni, M., \& Larsson, T. (2018). A model-driven decision arena: Augmenting decision making in early design. Proceedings of NordDesign: Design in the Era of Digitalization, NordDesign 2018.

Windheim, M. (2020). Decision-Making in Modular Product Family Design. In Cooperative Decision-Making in Modular Product Family Design (pp. 79-94). https://doi.org/10.1007/978-3-662-60715-2_4

Zavareh, M. T., Sadaune, S., Siedler, C., Aurich, J. C., \& Klaus, J. (2018). A Study on the sociotechnical aspects of digitization technologies for future integrated engineering work systems. 